\title{
CROWN LENGTHENING VS FORCED ERUPTION
}

\author{
Ayush Razdan Singh ${ }^{1}$, Ruchita Verma ${ }^{2}$ \\ ${ }^{1}$ Lecturer, ${ }^{2}$ P.G Student \\ Department of Conservative Dentistry \& Endodontics \\ Sardar Patel Post Graduate Institute of Dental and Medical Sciences, Lucknow \\ E-mail:dr_ayush_singh @ yahoo.com
}

\section{ABSTRACT}

Once the biologic width of the supporting periodontal attachment apparatus has been severely violated, more extensive procedures are often necessary to manage compromised root structure and supporting bone. Surgical techniques advocated are primarily corrective in nature and consist of root movement and repositioning or root removal and alteration of tooth morphology, with concomitant correction of the periodontium.

Some indications for surgical corrective intervention to manage radicular perforations include extensive cervical resorption or traumatic perforation which extends well below the osseous crest in both single and multirooted teeth and damage to the furcation region of multirooted teeth which is not responding to nonsurgical therapy, is not amenable to simple surgical correction, or is complicated and compromised further by extensive periodontal disease.

Common indications for orthodontic root extrusion include fractured tooth margins below crestal bone, deep carious margins, some isolated infrabony defects, and perforations from resorptions, post space preparation and aberrant access openings. When root extrusion is indicated to elevate a perforative root defect above the osseous crest; seldom is the desired result achieved without surgical crown lengthening. This is necessary to compensate for the coronal movement of the gingival attachment and alveolar bone which occurs with the tooth during eruption resulting in unacceptable esthetics. Although it is possible to extrude nearly any tooth the simplest cases are those that have single roots and an immediate proximal tooth on either side for appliance anchorage. Molars are generally difficult to treat as are the terminal teeth in the arch or free standing teeth.

Key words : Biologic width, crown lengthening, orthodontic root extrusion

\section{INTRODUCTION}

Aesthetic considerations have influenced the management of dental maladies in varying degrees for many years. Patient awareness and expectations have increased recently to the point that less than optimal aesthetics are no longer an acceptable outcome. In the $21^{\text {st }}$ century, the dental practitioner must be prepared to meet the challenges necessary to provide care that will result in a true condition of oral health. Current standards dictate the importance of avoiding procedures that will result in aesthetic compromise as well as the concept of providing patients with improved aesthetics whenever possible. An essential goal of treatment is long-term stability of the result; for this to be achieved the integrity of the dentogingival junction must be respected, and dental restorations and the periodontium must be in harmony. ${ }^{4}$

Extrusion is one of the techniques available for treating problems in the cervical region of the root that compromise biologic width. As first described by Heithersay and Ingber, this technique is based on osteophysiologic and orthodontic principles. ${ }^{11}$ Advantages of the traction technique presented here include ease, simplicity and low cost. A possible disadvantage is the need for a multidisciplinary approach. Depending on the technique selected, more than one professional might be involved in the procedure, which may increase cost. ${ }^{3}$ Extrusion is usually performed by means of fixed orthodontic appliances utilizing arch wires or elastics attached to the tooth but it can also be accomplished with the use of occlusal plates and elastics. ${ }^{?}$

Endodontic-orthodontic-periodontic cotreatment may be necessary to save teeth with advanced caries, traumatic destruction of the clinical crown, lateral root perforation, external or internal resorption near the alveolar crest or overzealous tooth preparation. Without such treatment these teeth may not offer sound tooth structure on which to place a restoration. ${ }^{14}$ 
Clinical crown lengthening refers to procedures designed to increase the extent of supragingival tooth structure for restorative or esthetic purpose. ${ }^{4}$ The need for clinical crown lengthening in the esthetic zone may not be related to restorative dental procedures. There are patients who present with excessive gingival display upon smiling (also referred to as "gummy smile") and the reduction of this excessive display is desirable for the purpose of improving esthetics. While there are several possible etiologies involved in excessive display of the gingival tissues upon smiling, cases in which teeth present with incomplete eruption (also referred to as altered passive eruption)

are most amenable to successful treatment with surgical crown lengthening. ${ }^{1}$ Isolated infrabony periodontal defects may also be amenable to crown lengthening . This will improve the existing periodontal environment by modifying the osseous topography and pocket epithelium as they relate to adjacent teeth. ${ }^{14}$ Carious invasion of the tooth in the cervical region can also result in a perforation, or near perforation, both laterally and in the furcation region, at or below the level of the crestal bone. Many of these require cases of crown lengthening, root extrusion and tooth resection to retain valuable radicular segments. ${ }^{5}$

\section{DISCUSSION}

Prior to developing a suitable treatment plan, it is essential to establish a complete and accurate assessment of conditions with which the patient presents. First, it is important to determine the chief complaint or the patient's reasons for seeking treatment.

These could include pain, swelling, impaired function, unsatisfactory aesthetics or a combination of these reasons. Next, the medical status of the patient must be reviewed and vital signs be recorded. This will determine the patient's suitability for dental treatment and identify any special precautions that must be taken. A common example of such precautions is premedication for the prevention of bacterial endocarditis. ${ }^{2}$

Selection of cases for extrusion is directly related to local factors, such as shape and length of the root, degree of compromise of biologic width, and the type of force and technique utilized. The term "forced eruption" might be erroneously interpreted if an excessive force is applied. In fact, the safety limit is not easy to establish, and the use of excessive force might be responsible for some root resorption. ${ }^{13}$ Retention is necessary to stop the tooth from returning to its original position, ${ }^{7}$ but recommendations for the retention period may vary. 15

Lengthening the clinical crown by removing supporting bone to expose sound tooth structure or eliminate the existing periodontal defect is done to fulfill the periodontal and occlusal requirements of a tooth in question. Forced orthodontic eruption in conjunction with endodontic, periodontal and restorative therapy is also an alternative. This multidisciplinary approach offers benefits not available with periodontal surgery alone (Figure 1). ${ }^{14}$

In health the alveolar bone mimics the rise and fall of the cementoenamel junction as it is followed around the tooth. The supra-alveolar tissue is comprised of sulcular epithelium, junctional epithelium and gingival connective tissue. When the margin of a restoration is being placed below the gingiva, it is of utmost importance to maintain the health and integrity of the biologic width. A restoration that impinges on the biologic width will result in progressive periodontal disease. The initial response to this insult may be gingival recession, edema or hyperplasia. ${ }^{14}$

To avoid periodontal pathosis, the dentist must maintain integrity of the biologic width. For this reason, an additional 1 or $2 \mathrm{~mm}$ of sound tooth structure must be available coronal to the epithelial attachment for placement of the margin of a restoration. The distance from the alveolar crest to the coronal extent of the remaining tooth structure should be a minimum of 3.5 to $4 \mathrm{~mm}$. With any less tooth structure the clinician risks impinging on the junctional epithelium and connective tissue attachment in a subgingival preparation. ${ }^{14}$

The biologic width of gingival tissue moves with the tooth as the tooth moves in health, maintaining its relationship to the tooth as the tooth is moved under controlled orthodontic force. In the presence of inflammatory periodontal disease, tooth movement may contribute to the deepening of an existing osseous defect. Therefore controlled orthodontic movement must take place in a periodontal environment that is as healthy as possible. It must be maintained in health throughout the movement phase of therapy. This is achieved through gentle but thorough scaling, root planning and soft tissue curettage on a biweekly basis as treatment progresses. Failure to control periodontal health during tooth movement may result in less than optimal treatment response. ${ }^{14}$ 
The traditional method of treating a tooth with advanced caries, resorption, or trauma extending apical or to the alveolar crest is to expose adequate sound tooth structure with periodontal surgery. In order to maintain a positive osseous architecture in which interradicular bone is coronal to radicular bone height, the operator must remove the supporting bone from adjacent teeth. The adjacent alveolar crest to the tooth in question must be scalloped to form a smooth, harmonious contour from tooth to tooth. This reduces alveolar support on teeth other than the one in question, increases the crown to root ratio of these teeth and compromises the esthetic appearance of anterior teeth (Figure 2). ${ }^{14}$

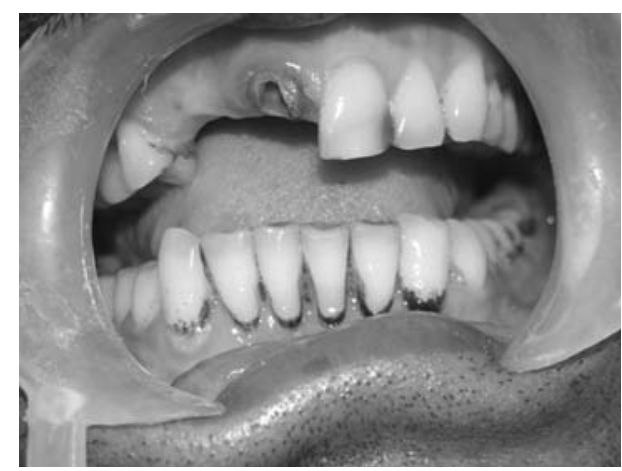

Figure 1 - Preoperative photograph of the patient

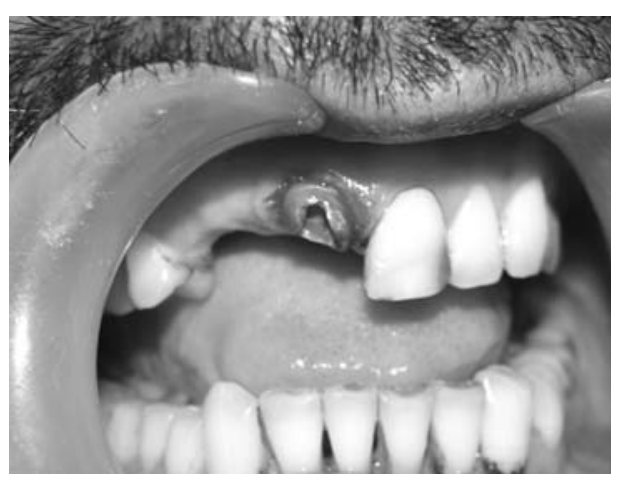

Figure 2 - Postoperative photograph of patient after crown lengthening

If the gingival margin of the tooth to be restored is in harmony with adjacent teeth and at an acceptable level with regard to aesthetics, then crown lengthening would need to be performed on all of the adjacent anterior teeth, and this could adversely affect aesthetics. In such cases, forced eruption combined with localized fibrotomy and thorough root planning or limited crown lengthening may be indicated. When a tooth is extruded, the resulting coronal-incisal height of the restoration that will be placed is less than if only a resective crown lengthening procedure were performed; consequently, the resulting crown-root ratio will be more favorable following extrusion than it would be with surgical crown lengthening alone. ${ }^{6}$

The scalloped gingival form associated with anterior teeth contributes to the difficulty in obtaining a cosmetic result when osseous surgery is completed on three or four teeth. The post operative appearance of the surgically corrected area will present open interdental embrasures and teeth that seem longer than their neighbors. ${ }^{14}$

In determining the suitability of a perforated root for extrusion, the final crown-root-ratio must be estimated. If a root is perforated completely below the crestal bone, the postoperative root length will be at least $3 \mathrm{~mm}$ less than the overall root length because of the allowance of $1 \mathrm{~mm}$ of clinical crown and $2 \mathrm{~mm}$ of gingival sulcus. Since most traumatic perforations tend to be oblique, rather than horizontal, which is more common with resorptive defects, the position of the perforation should be measured with a periodontal probe to determine the deepest apical extension. Effective root length will begin below this level. Root extrusion and crown lengthening may result in an unacceptably short root. Short roots will require, splinting to adjacent teeth and therefore in these cases, consideration should be given to possible extraction instead of extrusion. ${ }^{5}$

Orthodontically erupting the tooth with its attachment apparatus and gingival may eliminate the need for periodontal surgery to expose sound tooth structure and reduce alveolar support on adjacent teeth. Surgery may be necessary to level angular interdental alveolar crests created by tooth movement and reposition the overlying soft tissue to its proper coronal level. Augmentation of the gingival can be accomplished at the same surgery using soft tissue pedicle grafts of interproximal or edentulous ridge gingival. Periodontal surgery after eruption will restore form to the individual dental unit as it relates collectively to the adjacent teeth in the arch. ${ }^{14}$ The orthodontic extraction increases the width of the attached gingiva, and the mucogingival junction remains stable when the gingival margin migrates coronally. ${ }^{10}$

Exposing adequate sound tooth structure by periodontal surgery alone will lead to a shortened clinical root and a larger clinical crown as the tissues are positioned apically. The crown to root ratio of the tooth following surgery alone will exceed the crown- to -root ratio of the tooth that is first orthodontically erupted. Thus there is a relative improvement 
in the crown- to- root ratio of the tooth undergoing orthodontic eruption followed by periodontal therapy that does not occur after a surgical procedure alone. ${ }^{14}$

The end result of a forced eruption contributes to a more cosmetic and physiologic restoration. Osseous resection following forced eruption should be minimal on adjacent teeth. The position of the gingival tissue margin will approximate its original level following the surgical procedure, and a relative improvement in the crown to root ratio will be achieved. This reduces the dimension of the esthetic deformities created by surgical management alone. ${ }^{14}$

There are circumstances in which it may be desirable to have a longer clinical crown on a tooth than is present. If there is a sufficiently wide band of attached gingiva surrounding the tooth, this can be accomplished by gingivectomy using a diamond electrode. It is frequently necessary to do a second series of cuts to produce a bevel around the first. This will produce a better tissue contour without hard-to-clean edges near the tooth. This "bevel" also must be done only on attached gingiva. When surgery leaves an extensive post-operative wound, it is necessary to place a periodontal dressing which should be changed in 7 days. The lengthened tooth that results from this surgery should afford better retention for any crown placed on it, with margin placement in an area of the tooth more accessible for cleaning. If the band of attached gingiva is too narrow, it must be made wider with a graft or an alternative restoration must be made for the tooth. ${ }^{12}$

\section{CONCLUSION}

A final consideration in treatment planning extrusive procedures is the esthetics of root width, especially in the maxillary anterior region. Here roots taper significantly from cervical margin to the apex. In the case of an extruded root with a relatively uniform width, cervical width varies only slightly or does not change at all during extrusion. Therefore the width of the crown restoration will assume contours of the natural crown. With a markedly tapered root, the cervical diameter diminishes as the root is extruded and the crown restoration may have a distinct narrow dimension cervically, leaving excessively wide and unaesthetic embrasures or bulky, unphysiologic cervical crown margins. ${ }^{5}$

Harmony must exist between soft and hard tissue and between the periodontium of adjacent teeth. Often a combination of orthodontic extrusion and surgical crown lengthening can be employed to minimize the need for resective therapy on adjacent teeth, improve the crown-root ratio and facilitate a more aesthetic outcome. ${ }^{8}$

\section{ACKNOWLEDGEMENT}

The authors wish to acknowledge the contribution of Dr. Adarsh Kumar, Post Graduate Student, Final Year, Department of Conservative Dentistry \& Endodontics, Sardar Patel Post Graduate Institute of Dental \& Medical Sciences, Lucknow.

\section{REFERENCES}

1. Camargo PM, Melnick PR, Camargo LM. Clinical crown lengthening in esthetic zone. CDA Journal (2007); 35(7): 487-498.

2. Dajani AS, Taubert KA, Wilson W, Bolger AF, Bayer A, Ferrieri P, Gewitz MH, Shulman ST, Nouri S, Newburger JW, Hutto C, Pallasch TJ, Gage TW, Levison ME, Peter G, Zuccaro G Jr. Prevention of bacterial endocarditis. Recommendations by the American Heart Association. JAMA (1997); 277: 1794-1801.

3. Fellipe LA, Monteiro S, Vieira LC, Araujo E. Reestablishing biological width with forced eruption. Quintessence Int (2003); 34: 1-6.

4. Glossary of periodontal terms, the American Academy of Periodontology. 4th ed. Chicago (2001).

5. Gutmann JL, Harrison JW. Surgical endodontics. $1^{\text {st }}$ Edition. Ishiyaku EuroAmerica Inc, USA.

6. Ingber JS. Forced eruption: alteration of soft tissue cosmetic deformities. Int J Periodontics Restorative Dent (1989); 9: 416-425.

7. Johnson GK, Sivers JE. Forced eruption in crown-lengthening procedures. J Prosthet Dent (1986); 56: 424-427.

8. Jorgensen MG, Nowzari H. Aesthetic crown lengthening. Periodontology (2000); 27: 45-58

9. Mandel RC, Binzer WC, Withers JA. Forced eruption in restoring severely fractured teeth using removable orthodontic appliances. J Prosthet Dent (1982); 47: 269-274.

10. Nozawa T, Sugiyama T, Yamaguchi S, Ramos T, Komatsu S, Enomoto H. Buccal and coronal bone augmentation using forced eruption and buccal root torque: a case report. Int J Periodontics Restorative Dent (2003); 23(6):585-91.

11. Potashnick SR, Rosenberg ES. Forced eruption: principles in periodontics and restorative dentistry. J Prosthet Dent (1982); 48(2):141-8.

12. Shillingburg HT, Hobo S, Whitsett LD, Jacobi R, Brackett SE. Fundamentals of fixed prosthodontics. $3^{\text {rd }}$ Edition. Quitessence Publishing Co, New Delhi.

13. Stern N, Becker A. Forced eruption: Bilogical and clinical considerations. J Oral Rehabil (1980); 7: 395-402.

14. Weine FS. Endodontic therapy.5th Edition. Mosby Inc, USA.

15. Worth GB. Combined endodontic-orthodontic treatment of transverse root fractures in region of the alveolar crest. Oral Surg (1973); 36(3): 404-415. 